(C) 2009 IEEE. Personal use of this material is permitted. Permission from IEEE must be obtained for all other uses, in any current or future media, including reprinting/republishing this material for advertising or promotional purposes, creating new collective works, for resale or redistribution to servers or lists, or reuse of any copyrighted component of this work in other works. 


\title{
Frequency and Space Precoded MIMO OFDM with Substream Adaptation
}

\author{
Xiaojing Huang and Y. Jay Guo \\ Wireless Technologies Laboratory \\ CSIRO ICT Centre \\ Sydney, Australia \\ Xiaojing.Huang@csiro.au and Jay.Guo@csiro.au
}

\begin{abstract}
A new frequency and space precoding scheme for multiple input multiple output (MIMO) orthogonal frequency division multiplexing (OFDM) systems is presented. For frequency precoding, the data symbols to be transmitted are divided into multiple substreams, and a predefined unitary matrix is applied to each substream to obtain different linear combinations of data symbols in the substream to gain frequency diversity. For space precoding, different precoding matrices selected from a predefined orthogonal matrix are used to allocate each frequency precoded data symbol to all transmit antennas to gain spatial diversity. The number of substreams and the corresponding data symbol mapping scheme are also adaptively determined at the receiver under varying received signal strength and MIMO channel conditions, and are made available to the transmitter through a low-rate feedback channel. Simulation results show that the proposed MIMO OFDM system with adaptive substream selection can effectively exploit both frequency and spatial diversity, and deliver the maximum system throughput.
\end{abstract}

Keywords-Multiple input multiple output (MIMO), orthogonal frequency division multiplexing (OFDM), frequencyselective fading, frequency diversity, spatial diversity, and throughput.

\section{INTRODUCTION}

Multiple input multiple output (MIMO) technology uses multiple transmit and receive antennas in a wireless communication link to increase the system spectral efficiency and/or enhance the received radio signal quality. MIMO technology has attracted considerable research attention since its emerging in early 1990's, and is still evolving [1-3]. MIMO systems can operate in different modes, such as spatial diversity, spatial multiplexing, and beamforming, depending on the signal correlations among antenna elements [4-8].

Spatial diversity aims at improving the link quality, measured by the signal-to-noise ratio (SNR), between the transmitter and the receiver. For spatial multiplexing, multiple parallel data streams called substreams are transmitted simultaneously to increase the link capacity. For beamforming, the same data symbols are fed to all transmit antennas with appropriate complex weighting in order to maximize the SNR at the receiver. In order to achieve optimized system performance, it is necessary for a MIMO system to adaptively change its parameters, signal processing algorithms, or even physical antenna configurations according to different channel conditions and system requirements [9-14]. These adaptations often rely on a certain amount of channel state information (CSI) at the transmitter and/or the receiver. The CSI at the transmitter can be obtained from the receiver through a feedback channel. However, the large amount of channel state parameters, which grow with the product of the numbers of transmit and receive antennas and the number of multipaths, adds to the system complexity, and thus the feedback requirements can not be met if only a low-rate feedback channel is available [15].

MIMO systems are usually incorporated with the orthogonal frequency division multiplexing (OFDM) technique to cope with the frequency-selective fading [2, 16-17]. However, owing to the fact that OFDM converts a frequency-selective fading channel into a number of flat fading subchannels, the frequency diversity of an OFDM system is very poor. Channel coding has been traditionally used to gain diversity across frequency and time with the cost of reduced data rate [18-19]. Recently linear precoding or block spreading for OFDM has been proposed to improve the frequency diversity performance without data rate losses [20-26].

In order to improve the overall system spectral efficiency, i.e., the normalized throughput in bits per second per $\mathrm{Hz}$ (b/s/Hz), a MIMO OFDM system with combined frequency and space precoding is proposed in this paper. Similar to spatial multiplexing, the data symbols to be transmitted are first divided into substreams. However, the proposed system performs frequency precoding on each substream to obtain different linear combinations of the data symbols in the substream, which are then spread across the transmission frequency band through OFDM modulation in order to gain frequency diversity. After frequency precoding, the space precoding is followed to assign each frequency precoded symbol to all transmit antennas in order to achieve spatial diversity. The number of substreams and the corresponding data mapping scheme are adaptively selected based on the CSI through a very low-rate feedback channel. The selection of the space precoding matrix from a predefined orthogonal matrix also achieves certain beamforming effect. With the combined frequency and space precoding as well as substream adaptation, the proposed system is able to operate in a hybrid mode without explicit switching between the different MIMO modes, and thus can adapt itself to varying channel conditions and deliver optimized system performance.

The rest of the paper is organized as follows. In Section II, 
the frequency and space precoded MIMO OFDM system and the MIMO frequency-selective fading channel model are presented. In Section III, the minimum mean squared error (MMSE) equalization and detection method is derived, and the formulae for evaluating bit error rate (BER) and throughput are provided. Section IV explains the necessity for adaptive substream selection and describes the adaptation algorithm in order to achieve optimal throughput. Section V provides the simulation results to demonstrate the expected performance. Finally, conclusions are drawn in Section VI.

\section{SYSTEM MODELS}

The baseband model of the frequency and space precoded MIMO OFDM transmitter is shown in Fig. 1 (a), where a block of $M_{s} N$ input data symbols denoted as $x[i]$, $i=0,1, \cdots, M_{s} N-1$, is firstly divided through substream demultiplexing into $M_{s}$ groups of size $N$. Each group represents a data substream. In matrix form, the $M_{s}$ parallel substreams can be expressed as an $M_{s} \times N$ matrix

$$
\mathbf{X}=\left(\begin{array}{cccc}
x[0] & x[1] & \cdots & x[N-1] \\
x[N] & x[N+1] & \cdots & x[2 N-1] \\
\vdots & \vdots & \ddots & \vdots \\
x\left[\left(M_{s}-1\right) N\right] & x\left[\left(M_{s}-1\right) N+1\right] & \cdots & x\left[M_{s} N-1\right]
\end{array}\right) .
$$

It is also assumed that the data symbols $x[i]$ are mutually independent with average signal power $\sigma_{x}^{2}$.

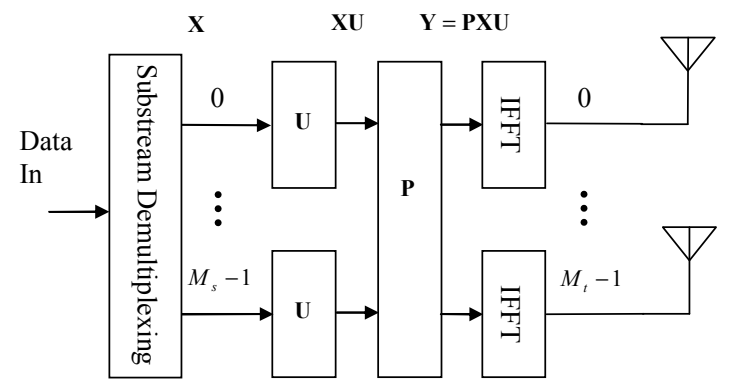

(a)

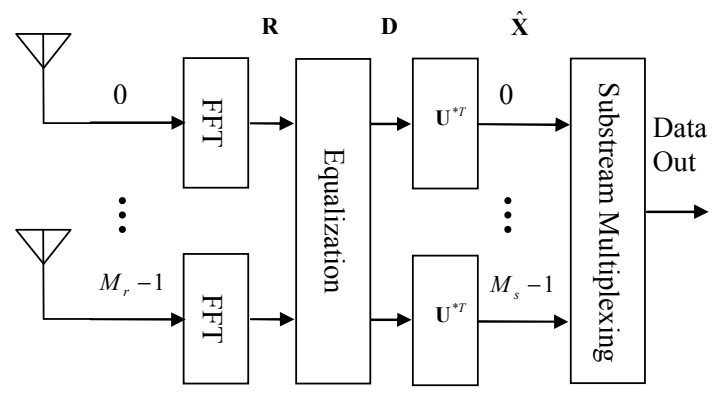

(b)

Fig. 1. Frequency and space precoded MIMO OFDM system: (a) transmitter and (b) receiver.
The frequency precoding is to apply a $N \times N$ unitary matrix $\mathbf{U}$ to each data substrream to produce a precoded data substream, and thus each precoded data symbol is a linear combination of the data symbols in the original substream. The precoding matrix $\mathbf{U}$ satisfies the property $\mathbf{U U}^{* T}=\mathbf{U}^{* T} \mathbf{U}=$ $\mathbf{U}^{*} \mathbf{U}^{T}=\mathbf{U}^{T} \mathbf{U}^{*}=\mathbf{I}_{N}$, where $(.)^{*}$ and $(.)^{T}$ denote the matrix conjugation and transposition respectively, and $\mathbf{I}_{N}$ is the identity matrix of order $N$. Applying this frequency precoding to all $M_{s}$ data substreams, the precoded data block can be expressed as $\mathbf{X U}$.

Suppose that the transmitter is equipped with $M_{t}$ transmit antennas. The space precoding is to apply an $M_{t} \times M_{s}$ matrix $\mathbf{P}$ to the $M_{s}$ frequency precoded data substreams to produce $M_{t}$ frequency and space precoded data substreams, expressed as $\mathbf{Y}=\mathbf{P X U}$ in matrix form, in order to be transmitted via the $M_{t}$ transmit antennas respectively. Each row of $\mathbf{Y}$ represents a frequency and space precoded data substream.

The space precoding matrix $\mathbf{P}$ is chosen as a subset of a predefined orthogonal matrix such as the Walsh-Hadamard matrix or discrete Fourier transform (DFT) matrix. However, the number of columns $M_{s}$, and the column vectors of $\mathbf{P}$ are determined based on the CSI obtained from the feedback channel. In addition, $\mathbf{P}$ should be scaled to satisfy the constraints: (1) $\|\mathbf{P}\|^{2}=1$, where $\|\cdot\|$ denotes the Frobenius norm, (2) $\left\|\mathbf{p}_{m}\right\|^{2}=1 / M_{t}$, where $\mathbf{p}_{m}$ is the $m$ th row of $\mathbf{P}$, and (3) $\left\|\mathbf{p}_{n}\right\|^{2}=1 / M_{s}$, where $\mathbf{p}_{n}$ is the $n$th column of $\mathbf{P}$. The first constraint ensures that the total power to be transmitted is unity. The second and third constraints ensure that the power is equally allocated to each transmit antenna and each substream respectively.

After performing the inverse fast Fourier transform (IFFT) of length $N$ to all precoded data substreams respectively, $M_{s}$ parallel time-domain signal substreams are generated. To avoid interference between adjacent OFDM symbols transmitted by a transmit antenna, either a cyclic prefix $(\mathrm{CP})$ or a zero-padded suffix (ZP) of sufficient length (longer than the maximum channel multipath delay $L$ in samples) needs to be added to each row of $\mathbf{Y}$ before transmission, but this is not shown in Fig. 1(a) for simplicity.

The receiver baseband model is shown in Fig. 1(b), where $M_{r}$ receive antennas are used. After removing the $\mathrm{CP}$ or performing an overlap-add operation (not shown) and taking the fast Fourier transform (FFT) for the signals received by all receive antennas respectively, the frequency-domain received signal can be represented by an $M_{r} \times N$ matrix $\mathbf{R}$. The $n$th column of $\mathbf{R}$ can be expressed as

$$
\mathbf{r}_{n}=\mathbf{H}[n] \mathbf{y}_{n}+\mathbf{v}_{n}, \quad n=0,1, \ldots, N-1
$$


where $\mathbf{y}_{n}$ is the $n$th column of the frequency and space precoded data matrix $\mathbf{Y}, \mathbf{v}_{n}$ is a zero-mean Gaussian noise vector with covariance matrix $E\left\{\mathbf{v}_{n} \mathbf{v}_{n}^{{ }^{*} T}\right\}=\sigma_{v}^{2} \mathbf{I}_{M_{r}}$, and $\mathbf{H}[n]$ is the $M_{r} \times M_{t}$ MIMO channel matrix at the subcarrier frequency indexed by $n$.

After converting the received signal into the frequencydomain by FFT, equalization and decoding are then followed to recover the data symbols in the $M_{s}$ data substreams which are finally multiplexed to produce a block of $M_{s} N$ output data symbols. Due to the complexity of the optimal maximum likelihood (ML) detection, MMSE equalization and decoding are applied, which will be described in the following section.

Now consider the frequency-selective MIMO channel with $L$ independent multipaths for any transmit-receive antenna pair [9]. The normalized frequency-domain MIMO channel matrices $\mathbf{H}[n]$ which ensure the total received signal power by any receive antenna to be unity can be modeled as

$$
\mathbf{H}[n]=\frac{1}{\sqrt{\operatorname{trace}\left\{\widetilde{\mathbf{R}}_{t}\right\} L}} \mathbf{R}_{r}^{\frac{1}{2}}\left(\sum_{l=0}^{L-1} \mathbf{H}_{w}[l] e^{-j \frac{2 \pi}{N} n l}\right) \mathbf{R}_{t}^{\frac{1}{2}}
$$

where $\mathbf{R}_{r}=\left(\rho_{r}^{|i-j|}\right)_{M_{r} \times M_{r}}$ and $\mathbf{R}_{t}=\left(\rho_{t}^{|i-j|}\right)_{M_{t} \times M_{t}}$ are the receive and transmit antenna correlation matrices respectively with $\rho_{r}$ and $\rho_{t}$ as the receive and transmit spatial correlation coefficients respectively, $\quad \widetilde{\mathbf{R}}_{t}=\mathbf{P}^{* T} \mathbf{R}_{t} \mathbf{P}$ is the equivalent transmit antenna correlation matrix after considering the space precoding, and $\mathbf{H}_{w}[l]$ are $M_{r} \times M_{t}$ random matrices with independent identically distributed (iid) zero mean and unity variance Gaussian random variables as their elements.

\section{MMSE EQUALIZATION AND DECODING}

To facilitate the MMSE equalization and detection, the set of the $N$ equations expressed by (2) can be combined and rewritten in matrix form as

$$
\begin{aligned}
\underline{\mathbf{R}} & =\operatorname{diag}\{\mathbf{H}[n]\} \underline{\mathbf{Y}}+\underline{\mathbf{V}} \\
& =\operatorname{diag}\{\mathbf{H}[n]\} \underline{\mathbf{P X} \mathbf{U}}+\underline{\mathbf{V}} \\
& =\operatorname{diag}\{\mathbf{H}[n]\}\left(\mathbf{U}^{T} \otimes \mathbf{P}\right) \underline{\mathbf{X}}+\underline{\mathbf{V}}
\end{aligned}
$$

where (.) denotes the stack form of a matrix (i.e., a column vector obtained by stacking all columns of a matrix), $\operatorname{diag}\{\mathbf{H}[n]\}$ denotes a block diagonal matrix with $\mathbf{H}[n]$, $n=0,1, \ldots, N-1$, as the diagonal blocks, and $\otimes$ denotes the Kronecker product. The property $\underline{\mathbf{A B C}}=\left(\mathbf{C}^{T} \otimes \mathbf{A}\right) \underline{\mathbf{B}}$ has been applied to derive (4).

From (4), an estimate of $\underline{\mathbf{X}}$ under the MMSE criterion can be obtained as

$$
\begin{aligned}
\underline{\hat{\mathbf{X}}}= & \left(\left(\operatorname{diag}\{\mathbf{H}[n]\}\left(\mathbf{U}^{T} \otimes \mathbf{P}\right)\right)^{* T} \operatorname{diag}\{\mathbf{H}[n]\}\left(\mathbf{U}^{T} \otimes \mathbf{P}\right)+\frac{1}{\gamma_{i n}} \mathbf{I}_{M_{s} N}\right)^{-1} \\
& \cdot\left(\operatorname{diag}\{\mathbf{H}[n]\}\left(\mathbf{U}^{T} \otimes \mathbf{P}\right)\right)^{* T} \underline{\mathbf{R}} \\
= & \left(\mathbf{U}^{*} \otimes \mathbf{I}_{M_{s}}\right) \operatorname{diag}\{\mathbf{F}[n]\} \underline{\mathbf{R}}
\end{aligned}
$$

where

$$
\mathbf{F}[n]=\left(\mathbf{P}^{{ }^{*} T} \mathbf{H}^{{ }^{T} T}[n] \mathbf{H}[n] \mathbf{P}+\frac{1}{\gamma_{i n}} \mathbf{I}_{M_{s}}\right)^{-1} \mathbf{P}^{{ }^{*} T} \mathbf{H}^{{ }^{T} T}[n]
$$

and $\gamma_{i n}=\frac{\sigma_{x}^{2}}{\sigma_{v}^{2}}$ is the input SNR before equalization. Eq. (5) and (6) suggest that the MMSE equalization and decoding should proceed in two steps. First, from the frequency-domain received signal matrix $\mathbf{R}$, we can obtain an equalized signal matrix $\mathbf{D}$ with its $n$th column calculated by $\mathbf{d}_{n}=\mathbf{F}[n] \mathbf{r}_{n}$. Second, since $\underline{\hat{\mathbf{X}}}$ can be expressed as $\left(\mathbf{U}^{*} \otimes \mathbf{I}_{M_{s}}\right) \underline{\mathbf{D}}$ from (5), we obtain the final detected data symbol matrix as $\hat{\mathbf{X}}=\mathbf{D} \mathbf{U}^{{ }^{*} T}$. This process is also illustrated in Section II Fig. 1 (b).

To analyze the system performance, the MMSE after solving (4) can be derived as

$$
\begin{aligned}
E\left\{(\underline{\hat{\mathbf{X}}}-\underline{\mathbf{X}})^{* T}(\underline{\hat{\mathbf{X}}}-\underline{\mathbf{X}})\right\} \\
\quad=\sigma_{x}^{2} \sum_{n=0}^{N-1} \operatorname{trace}\left\{\left(\gamma_{i n} \mathbf{P}^{* T} \mathbf{H}^{*_{T}}[n] \mathbf{H}[n] \mathbf{P}+\mathbf{I}_{M_{s}}\right)^{-1}\right\}
\end{aligned}
$$

and the output SNR is therefore

$$
\gamma=\frac{1}{\frac{1}{M_{s} N} \sum_{n=0}^{N-1} \operatorname{trace}\left\{\left(\gamma_{i n} \mathbf{P}^{{ }^{T}} \mathbf{H}^{{ }^{T} T}[n] \mathbf{H}[n] \mathbf{P}+\mathbf{I}_{M_{s}}\right)^{-1}\right\}}-1 .
$$

Assume that the $2^{k}$-ary ( $k$ is even) Gray-coded quadrature amplitude modulation (QAM) is used for data symbol mapping. When $k=2$, the mapping is the same as the quadrature phase shift keying (QPSK). The BER for a given channel realization is then expressed as [27]

$$
P_{e}=\frac{4}{k}\left(1-2^{-\frac{k}{2}}\right) Q\left(\sqrt{\frac{3}{2^{k}-1} \gamma}\right)
$$

where the Q-function is defined as $Q(x)=\frac{1}{\sqrt{2 \pi}} \int_{x}^{\infty} e^{-\frac{t^{2}}{2}} d t$. Also assume that the size of a data packet is $B$ in bits. The normalized throughput is thus given by

$$
T_{p}=M_{s} k\left(1-P_{e}\right)^{B}
$$

\section{ADAPTIVE SUBSTREAM SELECTION}

Since the ultimate goal of the proposed system is to achieve 
the maximum throughput, intuitively the number of substreams $M_{s}$ would be selected as large as possible from (10). However, it is observed from (8) that the output SNR $\gamma$ after MMSE equalization is also determined by $M_{s}$. To have a qualitative analysis on the relationship between $\gamma$ and $M_{s}$, we assume that the MIMO channel is perfect, i.e., all the transmit and receive antennas are independent and there is no multipath or fading, such that $\mathbf{H}^{{ }^{*} T}[n] \mathbf{H}[n] \rightarrow \frac{1}{\operatorname{trace}\left\{\mathbf{P}^{{ }^{*} T} \mathbf{P}\right\}} \mathbf{I}_{M_{t}}$. Then it can be proven that

$$
\gamma \rightarrow \frac{1}{M_{s}} \gamma_{\text {in }} \text {. }
$$

We see that, as $M_{s}$ increases, $\gamma$ decreases and hence the BER increases, which may in turn reduce the throughput. Therefore, the optimal number of substreams must be adaptively selected according to the different input SNRs and varying channel conditions.

Assume that $\gamma_{\text {in }}$ and $\mathbf{H}[n]$ can be perfectly estimated at the receiver. The procedure for adaptive substreams selection can be described as follows.

1. Set the initial $M_{s}$ to 1 ;

2. Select $M_{s}$ columns from a predefined $M_{t} \times M_{t}$ orthogonal matrix to compose the space precoding matrix $\mathbf{P}$;

3. Calculate $\gamma$ using (8), $P_{e}$ using (9), and hence $T_{p}$ using (10) under different QAM symbol mapping schemes. Determine the symbol mapping index $k$ which produces the maximum throughput;

4. Repeat step 2 and 3 for a different space precoding matrix $\mathbf{P}$ of $M_{s}$ columns and determine the best symbol mapping index $k$ until all possible $M_{s}$-column space precoding matrices are tried;

5. Increase $M_{s}$ by 1 , and repeat steps 2,3 , and 4 to determine the best space precoding matrix and symbol mapping index after all $M_{s}$ ranging from 1 to $M_{t}$ are tried.

Since the space precoding matrix is selected form a predefined orthogonal matrix, only the column indexes of the orthogonal matrix and the symbol mapping index are necessary to be fed back to the transmitter, which greatly reduces the CSI parameters. Otherwise, the CSI would be the $N M_{t} \times M_{s}$ matrices composed of the eigenvectors of $\mathbf{H}^{* T}[n] \mathbf{H}[n]$.

\section{Simulation Results}

The performance of the proposed frequency and space precoded MIMO OFDM system with adaptive substream selection is simulated and the results are provided in this section. The MIMO parameters are $M_{t}=M_{r}=4$ and $\rho_{t}=$ $\rho_{r}=0.1$ or 0.9 respectively. The OFDM parameters are $N=$ 64 and $L=16$. The space precoding matrix is chosen from a $4 \times 4$ Walsh-Hadamard matrix. The data symbol mappings used are $2^{k}$-ary QAM schemes for $k=2,4,6,8$, and 10 . At a given input SNR, a packet of size $B=8192$ bits is transmitted 10000 times with different channel realizations in order to calculate the average BER and normalized throughput.

Fig. 2 and Fig. 3 show the system average BERs using QPSK mapping and normalized throughputs versus input SNR respectively under different numbers of substreams with $\rho_{t}=$ $\rho_{r}=0.1$. The normalized throughput with adaptive substream selection is also shown in Fig. 3. We see that as the number of substreams increases the BER becomes worse as expected. More substreams can achieve greater throughput only at higher input SNRs. However, with adaptive substream selection, the best throughput can be always achieved.

Fig. 4 and Fig. 5 show the simulation results with the same performance measures as those used to produce Fig. 2 and Fig. 3 respectively but for $\rho_{t}=\rho_{r}=0.9$. We see that the transmit and receive antenna correlations significantly impact on the system performance. When the transmit antennas (and/or receive antennas) are more correlated, less substreams should be used. In the simulated examples, only one substream is sufficient to achieve the highest throughput in the considered input SNR range, even with adaptive substream selection (see Fig. 5 in which the throughput curve using adaptive substream selection coincides with that using one substream).

Comparing Fig. 2 with Fig. 4 (or Fig. 3 with Fig. 5), we see that the BER (or throughput) is improved significantly when the transmit and receive antennas are less correlated. This demonstrates that the proposed MIMO OFDM system is able to achieve spatial diversity.

Fig. 6 to Fig. 9 show the simulation results with the same performance measures as those used to produce Fig. 2 to Fig. 5 respectively but without frequency precoding. We see that the results in Fig. 2 to Fig. 5 are always better than those in Fig. 6 to Fig. 9 respectively. This confirms that the frequency precoding is necessary and effective to gain the frequency diversity provided by the frequency-selective fading channel.

The above simulation results clearly show that the optimal number of substreams depends on the received signal strength and transmit and/or receive antenna correlations. More substreams are only suitable at higher input SNR when the transmit and/or receive antennas are less correlated.

\section{CONCLUSIONS}

It has been demonstrated that, with adaptive substream selection, the proposed frequency and space precoded MIMO OFDM system can achieve both frequency diversity and spatial diversity, perform both spatial multiplexing and beamforming, and always deliver the optimum throughput. Since the frequency and space precoding matrices are both predefined, the channel state parameters can be significantly reduced, which allows for a very low-rate feedback channel to meet the CSI requirement for system adaptation. The multimode adaptive MIMO OFDM system is also well suited for practical implementation. 


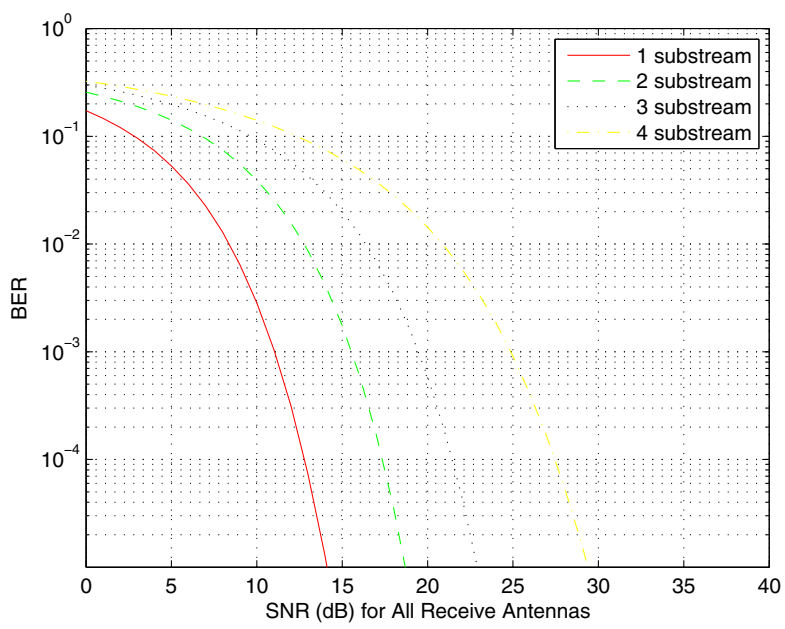

Fig. 2. Average BERs using QPSK mapping versus total input SNR for all receive antennas under different numbers of substreams $\left(\rho_{t}=\rho_{r}=0.1\right)$.

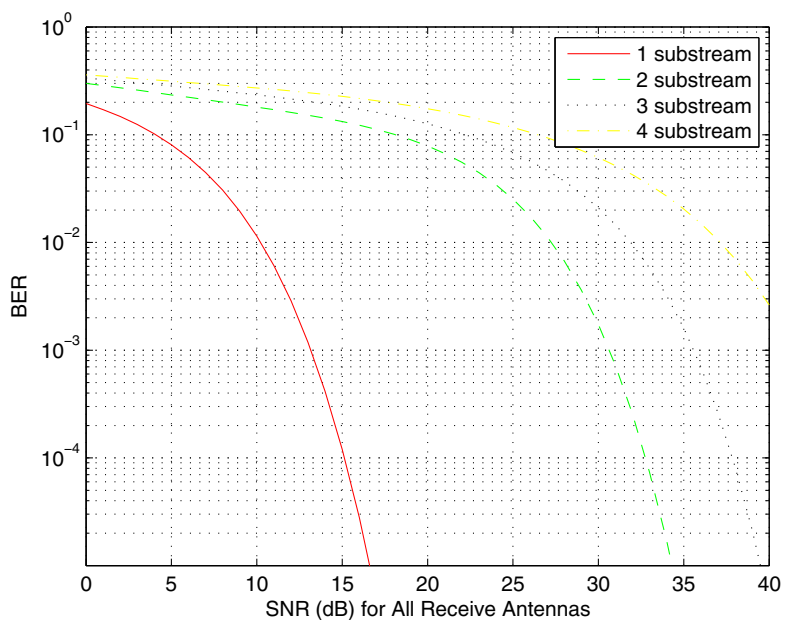

Fig. 4. Average BERs using QPSK mapping versus total input SNR for all receive antennas under different numbers of substreams $\left(\rho_{t}=\rho_{r}=0.9\right)$.

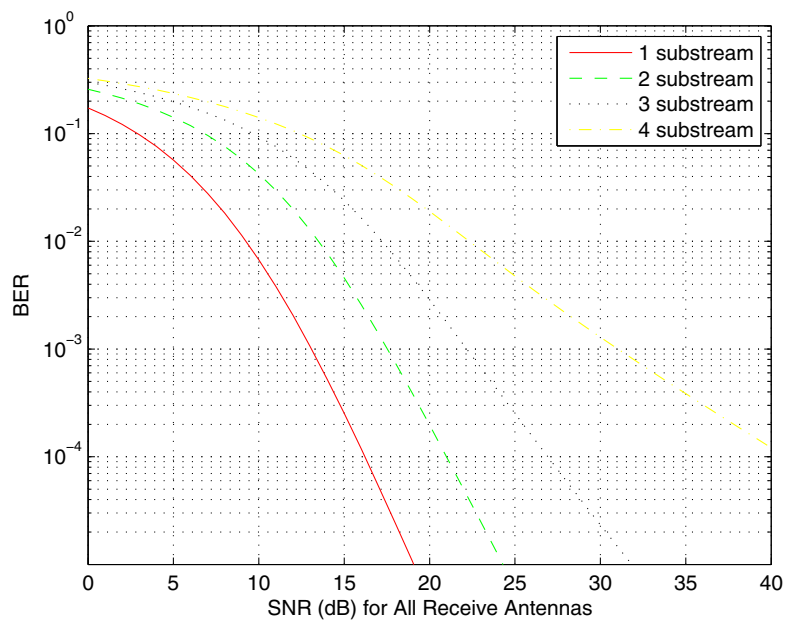

Fig. 6. Average BERs using QPSK mapping versus total input SNR for al receive antennas under different numbers of substreams without frequency precoding $\left(\rho_{t}=\rho_{r}=0.1\right)$.

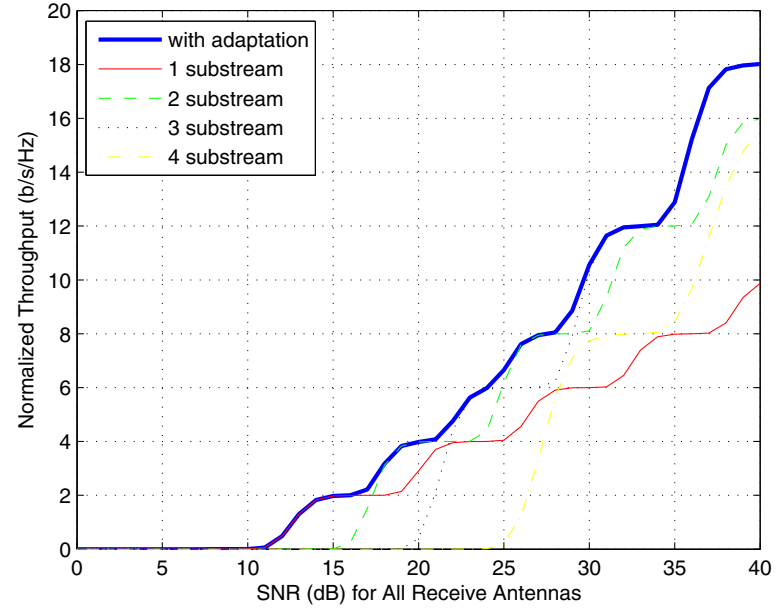

Fig. 3. Normalized throughputs versus total input SNR for all receive antennas under different numbers of substreams ( $\left.\rho_{t}=\rho_{r}=0.1\right)$.

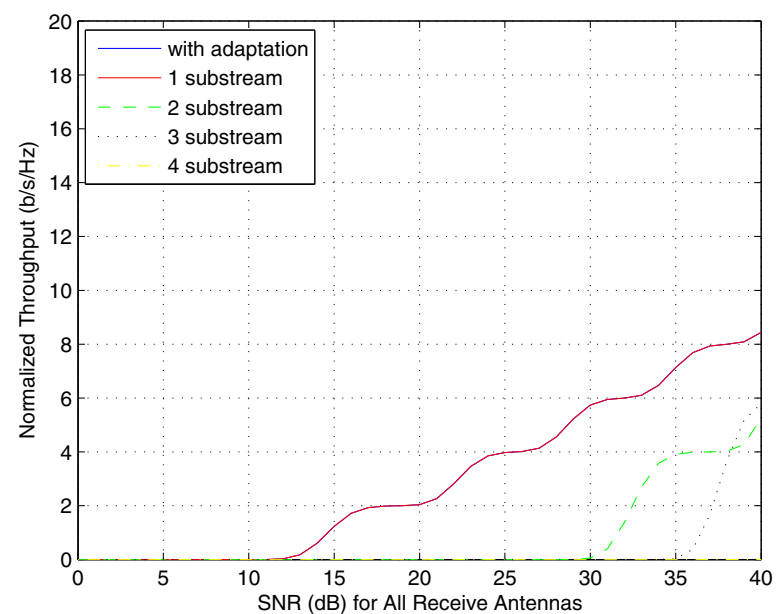

Fig. 5. Normalized throughputs versus total input SNR for all receive antennas under different numbers of substreams $\left(\rho_{t}=\rho_{r}=0.9\right)$.

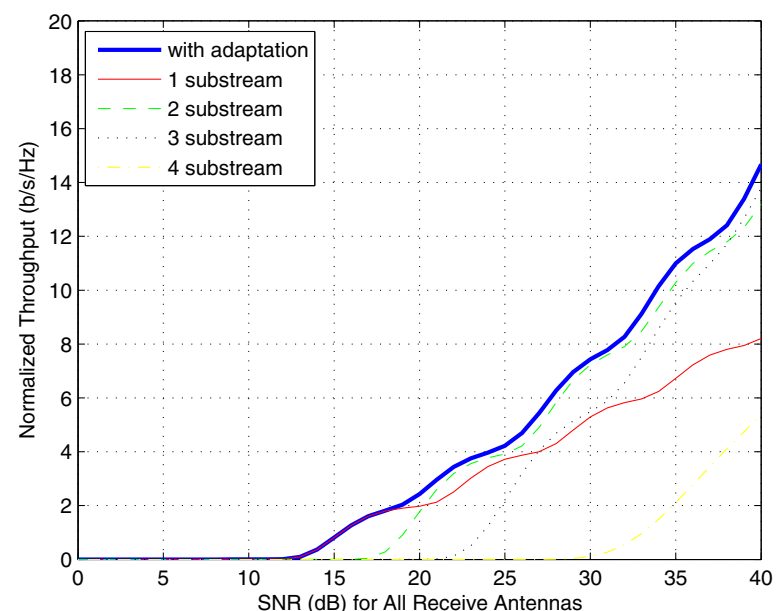

Fig. 7 Normalized throughputs versus total input SNR for all receive antennas under different numbers of substreams without frequency precoding ( $\rho_{t}=\rho_{r}$ $=0.1$. 


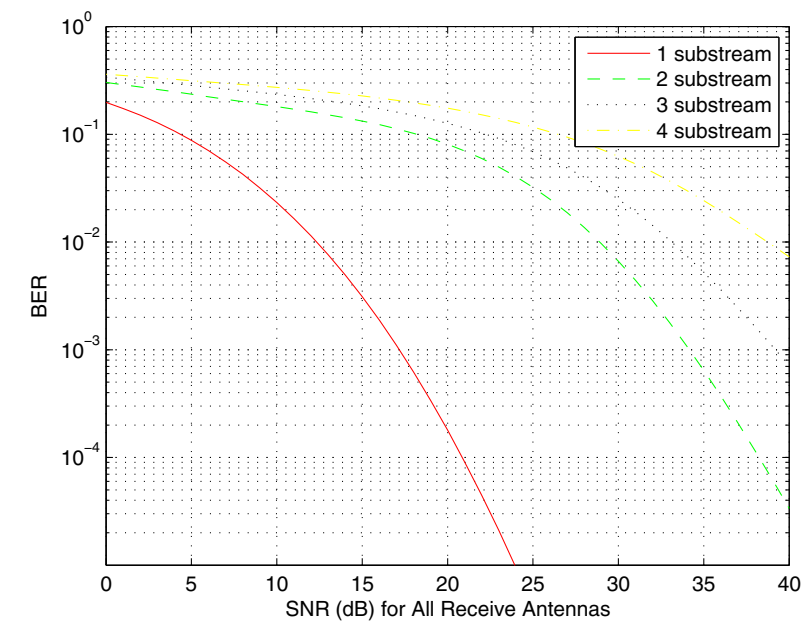

Fig. 8. Average BERs using QPSK mapping versus total input SNR for all receive antennas under different numbers of substreams without frequency $\operatorname{precoding}\left(\rho_{t}=\rho_{r}=0.9\right)$.

\section{REFERENCES}

[1] A. J. Paulraj and T. Kailath, "Increasing capacity in wireless broadcast systems using distributed transmission/directional reception," U.S. Paten 5345 599, 1994.

[2] A. J. Paulraj, D. A. Gore, R. U. Nabar, and H. Bölcskei, "An overview of MIMO communications - a key to gigabit wireless," invited paper, Proceedings of the IEEE, vol. 92, no. 2, February 2004, pp. 198-218.

[3] Y. J. Guo, Advances in Mobile Radio Access Networks, Artech House, Inc. 2004

[4] S. M. Alamouti, "A simple transmit diversity technique for wireless communications", IEEE Journal of Selected Areas in Communications, vol. 16, October 1998, pp. 1451-1458.

[5] A. Paulraj, R. Nabar, and D. Gore, Introduction to Space-Time Wireless Communications, Cambridge University Press, 2003.

[6] G. J. Foschini, "Layered space-time architecture for wireless communication in a fading environment when using multiple antennas," Bell Labs. Tech. J., vol. 1, no. 2, pp. 41-59, 1996.

[7] L. C. Godara, "Application of antenna arrays to mobile communications, part I: performance improvement, feasibility, and system considerations," Proceedings of the IEEE, vol. 85, no. 7, July 1997, pp. $1031-1060$.

[8] L. C. Godara, "Application of antenna arrays to mobile communications, part II: beamforming and direction of arrival consideration," Proceedings of the IEEE, vol. 85, August 1997, pp. 1195-1245.

[9] E. Yoon, J. Hansen, and A. Paulraj, "Space-frequency precoding with space-tap correlation information at the transmitter," IEEE Transactions on Communications, vol. 55, no. 9, September 2007, pp. 1702-1711.

[10] R. W. Heath, Jr. and D. J. Love, "Multimode antenna selection for spatial multiplexing systems with linear receivers," IEEE Transactions on Signal Processing, vol. 53, no. 8, August 2005, pp. 3042-3056.

[11] A. Dua, K. Medepalli, and A. J. Paulraj, "Receive antenna selection in MIMO systems using convex optimization," IEEE Transactions on Wireless Communications, vol. 5, no. 9, September 2006, pp. 2353-2357.

[12] R. W. Heath Jr., and A. J. Paulraj, "Switching between diversity and multiplexing in MIMO systems," IEEE Transactions on Communications, vol. 53, no. 6, June 2005, pp. 962-968.

[13] M. R. McKay, I. B. Collings, A. Forenza, and R. W. Heath, Jr., "Multiplexing/beamforming switching for coded MIMO in spatially correlated channels based on closed-form BER approximations," IEEE Transactions on Vehicular Technology, vol. 56, no 5, September 2007, pp. $2555-2567$.

[14] J. D. Boerman and J. T. Bernhard, "Performance study of pattern reconfigurable antennas in MIMO communication systems," IEEE Transactions on Antennas and Propagation, vol. 56, no. 1, January 2008, pp. 231-236.

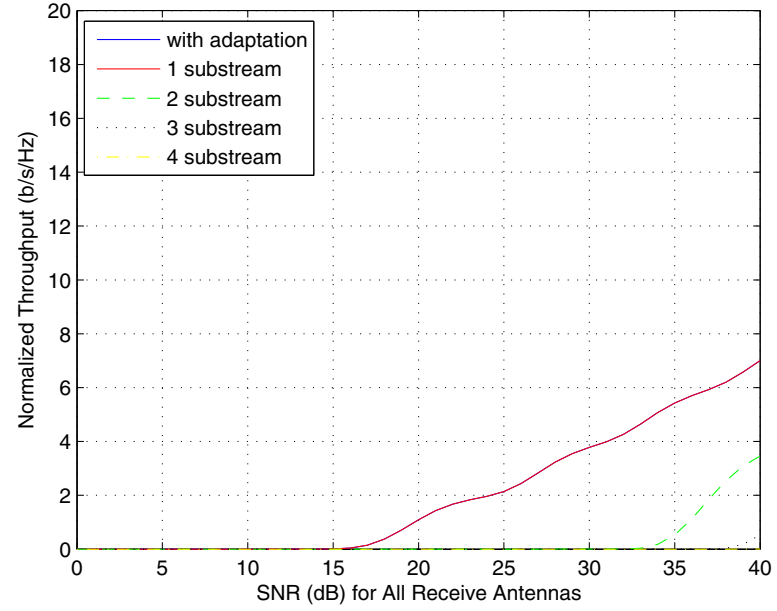

Fig. 9. Normalized throughputs versus total input SNR for all receive antennas under different numbers of substreams without frequency precoding ( $\rho_{t}=\rho_{r}$ $=0.9$ ).

[15] D. J. Love and R. W. Heath Jr.,"What is the value of limited feedback for MIMO channels?" IEEE Communications Magazine, October 2004, pp. 54-59.

[16] S. B. Weinstein and P. M. Ebert, "Data transmission by frequencydivision multiplexing using the discrete Fourier transform," IEEE Transactions on Communication Technology, COM-19, October 1971, pp. $628-634$.

[17] J. A. C. Bingham, "Multicarrier modulation for data transmission: An idea whose time has come," IEEE Communications Magazine, Vol. 28, May 1990, pp. 4-14.

[18] C. Berrou, A. Glavieux, and P. Thitimajshima, "Near Shannon limit error-correcting coding and decoding: Turbo-codes," in Proceedings of IEEE International Conference on Communications, Geneva, Switzerland, May 1993, Vol. 2, pp. 1064-1070.

[19] R. G. Gallager, "Low-density parity-check codes," IEEE Transactions on Information Theory, Vol. 8, No. 1, January 1962, pp. 21-28.

[20] Z. Wang and G. B. Giannakis, "Linearly precoded or coded OFDM against wireless channel fades?," in Proceedings of Signal Processing Advances in Wireless Communications Workshop, Taoyuan, Taiwan, March 20-23, 2001, pp. 267-270.

[21] Z. Liu, Y. Xin, and G. B. Giannakis, "Linear constellation precoding for OFDM with maximum multipath diversity and coding gains," IEEE Transactions on Communications, Vol. 51, No. 3, March 2003, pp. 416427.

[22] A. Bury, J. Egle, and J. Lindner, "Diversity comparison of spreading transforms for multicarrier spread spectrum transmission," IEEE Transactions on Communications, Vol. 51, No. 5, May 2003, pp. 774781.

[23] C. Tepedelenlioglu, "Maximum multipath diversity with linear equalization in precoded OFDM systems," IEEE Transactions on Information Theory, Vol. 50, No. 1, January 2004, pp. 232-235.

[24] M. L. McCloud, "Analysis and design of short block OFDM spreading matrices for use on multipath fading channels," IEEE Transactions on Communications, Vol. 53, No. 4, April 2005, pp. 656-665.

[25] K. I. Ahmed, C. Tepedelenlioglu, and A. Spanias, "Performance of precoded OFDM with channel estimation error," IEEE Transactions on Signal Processing, Vol. 54, No. 3, March 2006, pp. 1165-1171.

[26] X. Huang, "Multipath diversity of precoded OFDM with linear equalization," presented at the 2008 IEEE International Conference on Communications (ICC2008), Beijing, China, 19-23 May, 2008.

[27] J. Lu, K. B. Letaief, J. C-I. Chuang, and M. L. Lion, "M-PSK and MQAM BER computation using signal-space concepts," IEEE Transactions on Communications, Vol. 47, No. 2, February 1999, pp. 181-184. 